\title{
NORMALITY AND COMPACTNESS ARE EQUIVALENT IN HYPERSPACES
}

\author{
BY JAMES KEESLING
}

Communicated by Ernest Michael, October 31, 1969

Introduction. Let $X$ be a Hausdorff topological space and $2^{x}$ the space of all closed subsets of $X$ with the finite topology [5, Definition 1.7, p. 153] or [4]. This topology is also known as the exponential or Vietoris topology and $2^{x}$ is known as the hyperspace of $X$. It is known that $2^{x}$ is regular if and only if $2^{x}$ is completely regular if and only if $X$ is normal [5, Theorem 4.9, p. 163]. When is $2^{x}$ normal? It is clear that $2^{X}$ is normal if $X$ is compact for then $2^{x}$ is compact Hausdorff. Is the converse true? Ivanova has shown in [2] that if $X$ is a well ordered space with the order topology, then $2^{x}$ normal implies that $X$ is compact. In [3] the author has shown that $2^{2^{X}}$ is normal if and only if $X$ is compact. The purpose of this announcement is to indicate that, assuming $\mathrm{CH}$ (the continuum hypothesis), $2^{x}$ normal implies that $X$ is compact.

1. A compactification result. Let $F_{n}(X)$ be the subset of $2^{x}$ consisting of those subsets of $X$ having cardinality at most $n$. Let $F_{n}(X)$ have the topology it inherits from $2^{X}$. A corollary to $[1$, Theorem 1 , p. 371] is that for $n \geqq 2$ and $X$ completely regular, $\beta\left(X^{n}\right)=(\beta X)^{n}$ if and only if $X^{n}$ is pseudocompact. Using this result we show that for $X$ completely regular, there is a corresponding result for $\mathfrak{F}_{n}(X)$.

Theorem 1. Let $n \geqq 2$. If $X$ is completely regular, then $\beta \Im_{n}(X)$ $=F_{n}(\beta X)$ if and only if $\mathfrak{F}_{n}(X)$ is pseudocompact if and only if $X^{n}$ is pseudocompact.

This result plays an important role in our study of the normality of hyperspaces.

2. The normality of hyperspaces. The proof of the next proposition makes strong use of Theorem 1.

Proposition. If $2^{X}$ is normal, then $X$ is countably compact and normal. If in addition $X$ is not compact, then there is an $n$ such that $X^{n}$ is not pseudocompact.

AMS Subject Classifications. Primary 5423, 5425, 5465; Secondary 5453, 5456.

Key Words and Phrases. Hyperspaces, finite topology, Vietoris topology, exponential topology, normality, symmetric product spaces, pseudocompactness, Stone-Čech compactification, compactification of symmetric products. 
It is not known whether $X$ normal and countably compact implies that $X^{n}$ is pseudocompact for all $n$. If this should happen to be true, then the proposition immediately gives us that $2^{X}$ normal implies $X$ compact without the aid of $\mathrm{CH}$.

LEммA. If $X$ is a countably compact $k$-space, then $X^{n}$ is countably compact for all $n$.

Actually, a more general result is true. If $X$ is a countably compact $k$-space and $Y$ is countably compact, then $X \times Y$ is countably compact. The lemma together with the proposition gives us the next theorem.

Theorem 2. If $X$ is a $k$-space, then $2^{X}$ normal implies that $X$ is compact.

The next theorem is our strongest result. To obtain it, it was necessary to assume $\mathrm{CH}$. Using the proposition we were able to show that if there is a noncompact space $X$ such that $2^{X}$ is normal, then there is a separable space $Y$ which is noncompact such that $2^{Y}$ is also normal. With the aid of $\mathrm{CH}$ we were able to show that for $X$ separable, $2^{X}$ normal implies that $X$ is compact. In the proof we used some imbedding techniques developed in [3] and a theorem of Tamano on the normality of product spaces [6].

Theorem 3. Assume CH. Then $2^{X}$ is normal if and only if $X$ is compact.

The details will appear elsewhere.

\section{REFERENCES}

1. I. Glicksberg, Stone-Čech compactifications of products, Trans. Amer. Math. Soc. 90 (1959), 369-382. MR 21 \#4405.

2. V. M. Ivanova, On the theory of spaces of subsets, Dokl. Akad. Nauk SSSR 101 (1955), 601-603. (Russian) MR 16, 1041.

3. J. Keesling, Normality and properties related to compactness in hyperspaces, Proc. Amer. Math. Soc. 24 (1970), 760-766.

4. K. Kuratowski, Topologie. Vol. I, PWN, Warsaw, 1958; English transl., Academic Press, New York, and PWN, Warsaw, 1966, pp. 160-182. MR 19, 873; MR 36 \#839; 840.

5. E. Michael, Topologies on spaces of subsets, Trans. Amer. Math. Soc. 71 (1951), 152-182. MR 13, 54.

6. H. Tamano, On paracompactness, Pacific J. Math. 10 (1960), 1043-1047. MR 23 \#A2186.

University of Florida, Gainesville, Florida 32601 